\title{
Optimization of LEACH Protocol and Environmental Monitor System Design based on WSN
}

\author{
Xiajun Ding ${ }^{1}$, Xiaodan Jiang ${ }^{2}$, Lu zhang $^{3}$ and Shuangxia Han ${ }^{4}$ \\ 1,2,3,4 College of Electrical and Information Engineering, Quzhou Collegey Quzhou, \\ Zhejiang, China

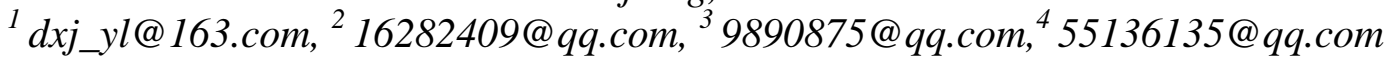

\begin{abstract}
In wireless sensor networks (WSN), sensor nodes just can use battery as their power resource and the power is quite limited. So, energy efficiency research is the key issue in design reasonable wireless sensor network routing protocols. In the paper, a revised cluster election method named LEACH-PSOv has been proposed to strengthen the hierarchical routing protocol low energy adaptive clustering hierarchy (LEACH). In the algorithm, particle colony optimization has been used to divide the network into more than one sub clusters and in each sub clusters head node and vice-head node would be elected to transfer the information to sink node. The algorithm proposed can be effectively to extend the lifetime of the networks. The simulation results show that the life time with new algorithm can extend the lifetime. Based on the improved LEACH algorithm, environmental monitor system has been designed.
\end{abstract}

Keywords: wireless sensor network, LEACH, PSOv, cluster

\section{Introduction}

A wireless sensor network (WSN) consists of many distributed sensors to monitor physical or environmental conditions, such as temperature, sound, pressure, etc. and to cooperatively pass their collected information through the network to a main location. The networks can be used in many applications. The WSN is composed of many nodes, where each node can connect to other sensors. Environmental Sensor Networks has been used in the environment research [1], such as volcanoes [2], oceans [3], glaciers [4], etc. There are various conditions that WSN used in monitoring, data analysis and data mining [5].

Many communication protocols for WSN have been developed. While main research was concentrate on energy efficiency, wireless link reliability [6], real-time capabilities [7], or quality-of-service. All research is considered to the variety of future applications to replace or enhance the conventional wire-based networks. But for the reason of sensor nodes just can use battery as their power resource in WSN, and the power is quite limited. So, energy efficiency research is the key issue in design reasonable wireless sensor network routing protocols.

Many methods and algorithms have been developed and most of them are based on low energy adaptive clustering hierarchy LEACH and some other algorithms, such as improved LEACH [8], LEACH-SM [9], TB-LEACH [10], V-LEACH [11], NDEA [12], energy balanced based routing protocol [13], TL-LEACH and DD-LEACH [14], improved LEACH [15-17], LEACH-HPR [18], etc. Due to the hazardous working conditions, un-replaced and un-recharged battery would be consumed rapidly, so the research of energy efficiency is an 
endless research, and more and more method to extend the lifetime of the nodes or networks would be proposed.

In this paper, a improved method, LEACH-PSOv, has been proposed to extend the lifetime of nodes and networks, and based on the algorithm environment monitoring system has been designed. The remainder of the paper is organized as follows: LEACH algorithm would be revisited in Section 2. The LEACH-PSOv and the simulation would be expressed in Section 3. Environment monitoring system design would be designed in Section 4 and the following is conclusion in Section 5.

\section{LEACH}

LEACH protocol is a kind of algorithm. In the network, the nodes would be organized by cluster. In the cluster, a node would be determined as the cluster head [19]. The process is organized in periodical manner, and each round would be divided into two steps: cluster building step and stable data communication step. In the first step, close nodes would make a cluster dynamically, and one node would be chosen to be the cluster head, which collects, processes and send information to a sink node. In the second step, each node in the cluster would sent message to the cluster head, and then the head would deal with and send it. In the process, head node would collect and fuse information and send it to sink node, so it would consume more energy than other nodes. Leach algorithm could meet the demand that each node in one cluster would have equal possibility to be the head node to balance the exist energy. LEACH protocol cluster structure is showed in Figure 1.

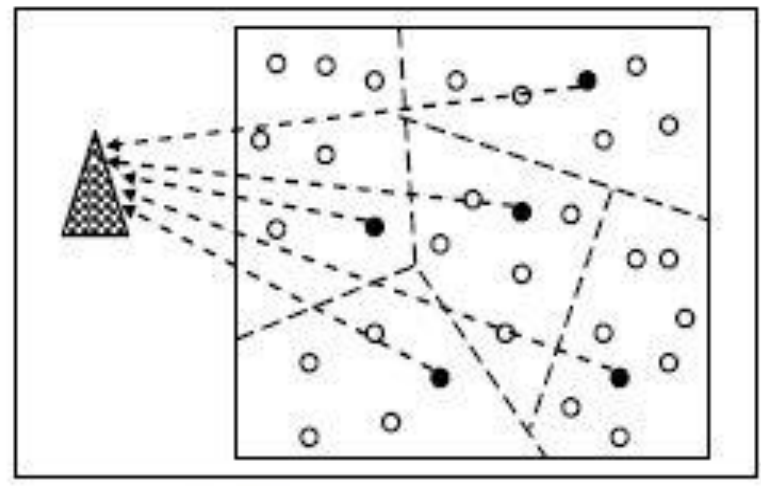

Figure 1. LEACH Protocol Cluster Structure

The election method of head node in LEACH would be described as following: (1) every node would produce a random number between 0 and 1 , and if the number is less than a predefined value of $T(n)$, then it would be elected as cluster head and send pronunciation to other nodes, and if the node has been the cluster node before, and the $T(n)$ would be set to 0 . It means that the node cannot be head again. If a node has not been the head node before, the probability of being selected is $T(n) \cdot T(n)$ would increase with the number of being head node increases. So the nodes, which have not been head node before would have a bigger probability. When just a node of not being elected as cluster node has left, $T(n)$ of the node would be set as 1 . That means the node would be cluster head. $T(n)$ 


$$
T(n)=\left\{\begin{array}{cc}
\frac{p}{1-p(r \bmod (1 / p))} & \text { if }(n \in G) \\
0 & \text { otherwise }
\end{array}\right.
$$

Where, $p$ means the probability of the number of cluster head in all nodes of the cluster; $r$ is the number of the current round, $G$ is the nodes which have not been the head node before. After the election of the head node, the head node would send information to perform to other nodes. And other nodes would join different clusters dynamically base on the distance and some other index. When all the nodes joins the clusters, they would send message to the cluster head and the cluster head would produce the time message TDMA to inform all the nodes in the clusters. Due to keep the nodes in one clusters to join another, the head node would also send CDMA code at the same time. Each node would send message to the head node in time-interval after they receive the TDMA and CDMA code. When the transmission is over, cluster head would collect and process the data and results would be sent to sink node and the round would be going into next.

There are also two level hierarchical structure LEACH. In the structure, first level is cluster sand the second level is nodes. In the cluster, head node would be chosen and in the network, head cluster would be elected to send message to base station.

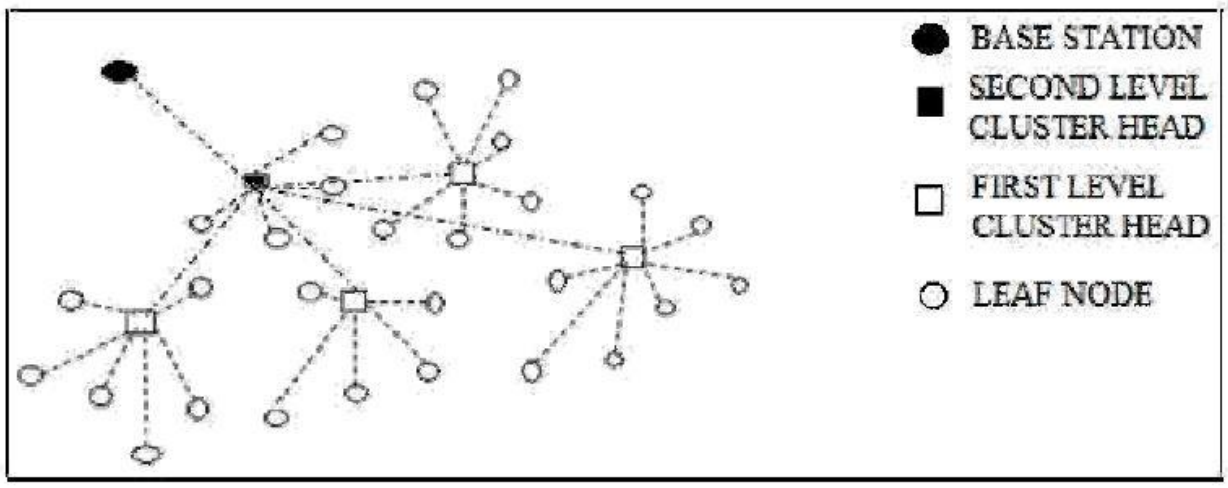

Figure 2. Two Level Hierarchical Structure

\section{LEACH-PSOv}

The improved algorithm is based on PSO and vice-cluster head. Firstly, the whole network would be divided into some sub clusters and secondly, the cluster head and vice cluster head would be elected based on the information of energy. After the two steps, the algorithm is accomplished.

\subsection{PSO}

Particle swarm optimization (PSO) is a computational optimization method to solve the problems by iteratively to acquire the solution at good quality. PSO optimizes problems according to a series of candidate solutions. PSO is originally proposed by Kennedy, Eberhart and Shi $[19,20]$. Some survey of PSO applications is made by Poli [21]. PSO can search very large spaces of candidate solutions without any assumptions. PSO used in multi-objective has been widely studied [23-25]. So it is very convenient to use in WSN to develop the LEACH protocol in balance the energy distribution in different area of the network. 


\subsection{Improved LEACH-PSOv}

\subsubsection{Division of Network}

It is assumed that there are $\mathrm{N}$ nodes in the network, and would be divided into $\mathrm{M}$ clusters, that is to say there would be $N / M$ nodes in each clusters. Firstly, to construct a splitter to divided the whole network into two domains with same nodes, and the splitter would be described as:

$$
U=(x, y, \theta)
$$

Where, $(x, y)$ is location on the splitter of nodes, $\theta$ is the angular between the splitter and $\mathrm{X}$ axle.

Define the function fitness as the following:

$$
\text { fitness }=\left(c_{1}-f_{1} N\right)^{2}+\left(c_{2}-f_{2} \mathrm{~N}\right)^{2}
$$

Where, $c_{i}(i=1,2)$ is the number of nodes in domain $i . f_{i}$ would be determined as the following:

$$
f_{i}=\frac{M_{i}}{M}
$$

The $i=(1,2)$

Where, $m_{i}$ is the expectation of number of cluster nodes in domain $i$.

Then, the division is finished.

The algorithm of cluster division would be divided into some steps:

(1) All nodes in the network send information of message (including location and energy information) to sink node. Sink node would divide the network into clusters after received the message and define $Q$ particles;

(2) Set random number to parameters of $x, y, \theta$, and construct the splitter. The whole network would be divided in to $Q \times 2$ clusters. Due to the location of nodes in the network are known, $c_{i}(i=1,2)$ of each node would be determined to calculate the values of fitness .

(3) Compare the value of fitness with minimum fitness value in the last round, the less one would be elected as a general extrum $P_{g d}$; Compare the fitness value of individual node, the least would be elected as individual extrum $P_{i d}$, and update the value of $x, y, \theta$ :

$$
\begin{aligned}
& X_{x i d}=X_{x i d}+V_{x i d} \\
& X_{y i d}=X_{y i d}+V_{y i d} \\
& X_{\theta i d}=X_{\theta i d}+V_{\theta i d}
\end{aligned}
$$

Where, $x_{y i d}, X_{y i d}$ represent the location of particle; $x_{\text {oid }}$ is the angular of the splitter;

$V_{x i d}, V_{y i d}, V_{\theta i d}$ are the search speed in three dimensions, and they would be determined as following:

$$
\begin{gathered}
v_{x i d}=\omega v_{x i d}+c_{1} \times \operatorname{rand}()\left(P_{i d}-X_{x i d}\right)+c_{2} \times \operatorname{rand}()\left(P_{g d}-X_{x i d}\right) \\
v_{y i d}=\omega v_{y i d}+c_{1} \times \operatorname{rand}()\left(P_{i d}-X_{y i d}\right)+c_{2} \times \operatorname{rand}()\left(P_{g d}-X_{y i d}\right) \\
v_{\theta i d}=\omega v_{\theta i d}+c_{1} \times \operatorname{rand}()\left(P_{i d}-X_{\theta i d}\right)+c_{2} \times \operatorname{rand}()\left(P_{g d}-X_{\theta i d}\right)
\end{gathered}
$$

Where, $c_{1}, c_{2}$ are the study factor, and $c_{1}=c_{2}=2 ; \operatorname{rand}()$ is random number between 0 and 1 ; $\omega$ is the weighted factor.

(4) After the update of $x, y, \theta$, go to step 2 to continue the research process. When the value of fitness is 0 or equal to the maximum times of search, the process would finished. Ideally, if 
the value of fitness is approximately 0 , and the whole network would be divided into two clusters with equal nodes.

(5) Use the method of above to divide the clusters, until get the demanded number of clusters.

\subsubsection{Election of Cluster Head and Vice Cluster Head}

After we get the clusters with equal nodes, cluster head and vice cluster head would be elected. In order to balance the energy of each nodes in the clusters, nodes with more existing energy would have higher probability to be the cluster head and vice cluster head. Firstly, a energy threshold $\eta$ would be set. Secondly, nodes in clusters would be classified into two sets: higher energy nodes (existing energy is bigger than $\eta$ ) and lower energy nodes (existing energy is less than $\eta$ ). Percentage of higher energy nodes in all nodes can be acquired based on the calculation and set $\alpha$ as the difference between energy of higher energy nodes and lower energy nodes. Define $P_{\text {hen }}$ and $P_{t e n}$ are the probability of higher energy nodes and lower energy nodes to be cluster head respectively.

$$
\begin{gathered}
P_{l e n}=\frac{p}{1+\alpha \cdot m} \\
P_{h e n}=\frac{p}{1+\alpha \cdot m} \times(1+\alpha)
\end{gathered}
$$

We use $P_{\text {hen }}$ and $P_{\text {ten }}$ as $P$ in the calculation method of $T(n)$ described before, then we can know:

$$
T\left(s_{\text {len }}\right)=\left\{\begin{array}{cc}
\frac{p_{l e n}}{1-p_{l e n}\left(r \bmod \left(1 / p_{l e n}\right)\right)} & \text { if }(n \in G) \\
0 & \text { otherwise }
\end{array}\right.
$$

Where, $r$ is the current round; ${ }_{G}$ is the lower energy nodes which have not been the head node before; and $T_{\left(s_{t e n}\right)}$ is the threshold used for lower energy nodes.

The same as lower energy nodes, $T\left(s_{\text {hen }}\right)$ can also be acquired as the following:

$$
T\left(s_{\text {hen }}\right)=\left\{\begin{array}{cc}
\frac{p_{\text {hen }}}{1-p_{\text {hen }}\left(r \bmod \left(1 / p_{\text {hen }}\right)\right)} & \text { if }(n \in G) \\
0 & \text { otherwise }
\end{array}\right.
$$

Where, ${ }_{G}$ is the higher energy nodes which have not been the head node before; ${ }_{T\left(s_{\text {hen }}\right)}$ is the threshold used for lower energy nodes.

As described before, we can get cluster head node in each cluster. After the election of cluster head, the higher energy node, which has the greatest distance with the cluster head, would be elected as vice cluster head. When the first round is finished, compare the existing energy of cluster head and vice cluster head, and the node with higher energy would be elected as cluster head to go on the second round. In the second round, there would be no vice cluster head.

When the cluster head and vice cluster head have been elected, the network go into data transmission period. Process in this period is the same as LEACH. 


\subsection{Simulation and Verification}

The simulation tool is MATLAB (version 7.10), which is very effective to simulate and compare the performance of the LEACH and LEACH-PSOv algorithm. The random, 100node network shown in Figure 3; the BS was placed at location $(50,50)$. The test network parameters are shown in Table 1.

Table 1. Input Parameter

\begin{tabular}{|c|c|}
\hline Parameter & variables \\
\hline Node & 100 \\
\hline Network Size & $100 \mathrm{~m}^{*} 100 \mathrm{~m}$ \\
\hline Base station location & $(50,50)$ \\
\hline Node energy & $0.5 \mathrm{j}$ \\
\hline Node position & Based on network topology \\
\hline No of rounds executed & 5000 \\
\hline Energy dissipation for transmission & $5 \mathrm{E}-8 \mathrm{j} / \mathrm{bit}$ \\
\hline Energy dissipation for reception \\
\hline $\begin{array}{c}\text { The probability that a node can be } \\
\text { cluster head }\end{array}$ & 0.2 \\
\hline Data aggregation energy & $5 \mathrm{E}-9 \mathrm{j} / \mathrm{bit}$ \\
\hline
\end{tabular}

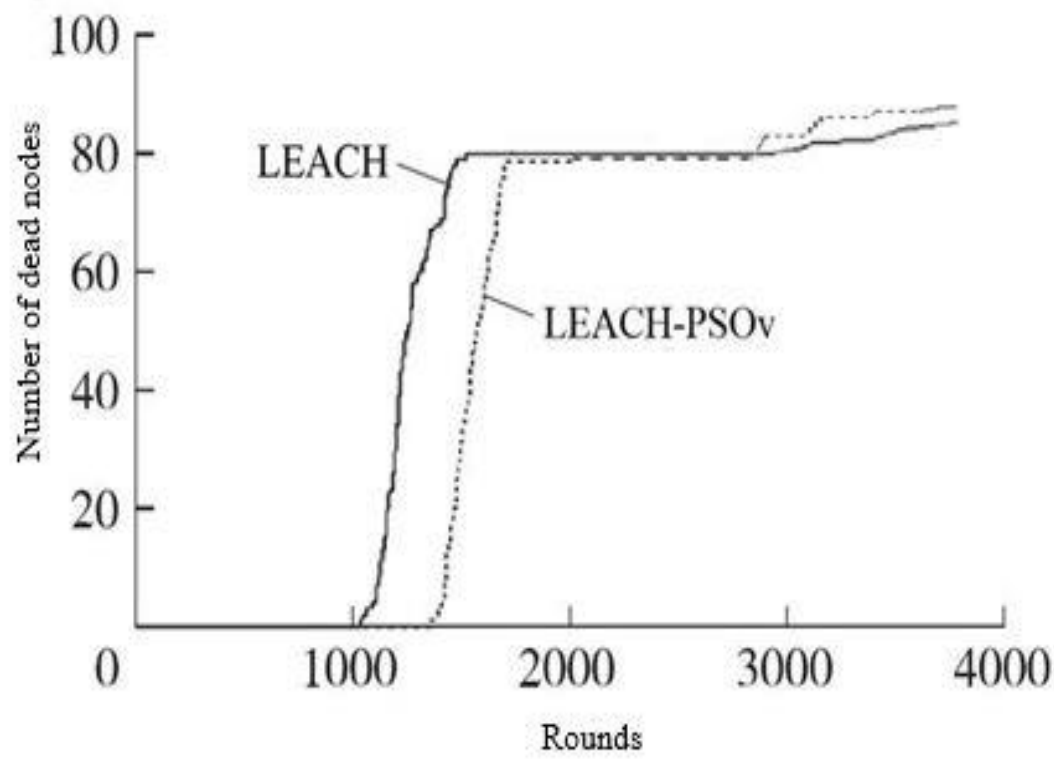

Figure 4. Death Nodes in both LEACH and LEACH-PSOv Algorithm

Figure 4 shows the veriation of dead nodes in the network with both algorithms. From the figure we can see that first dead node with LEACH and LEACH-PSOv algorithm appeared at 1054th and 1332nd round respectively. The LEACH-PSOv algorithem extends nearly 33\% additional time with no node death. In both algorithm, vast amount of nodes died after 1700 rounds. In LEACH algorithm, due to the head node is produced by random number in the whole network, it may lead the concentration of cluster head nodes and take more energy to transmit the message from normal nodes to the head nods. In LEACH-PSOv algorithm, the 
whole network would be divided into several sub cluters. This method can reduce the overlap of covered area of each cluster, and the cluster head node election is based on the existing energy to reduce the probability of lower energy node to be head node. Because of energy balance of each node, the network can operates a longer time with no nodes death. This means that the whole network performance is greatly improved, and because of no node died in the first 1300 rounds, the efficiency of the network is higher in LEACH-PSOv algorithm.

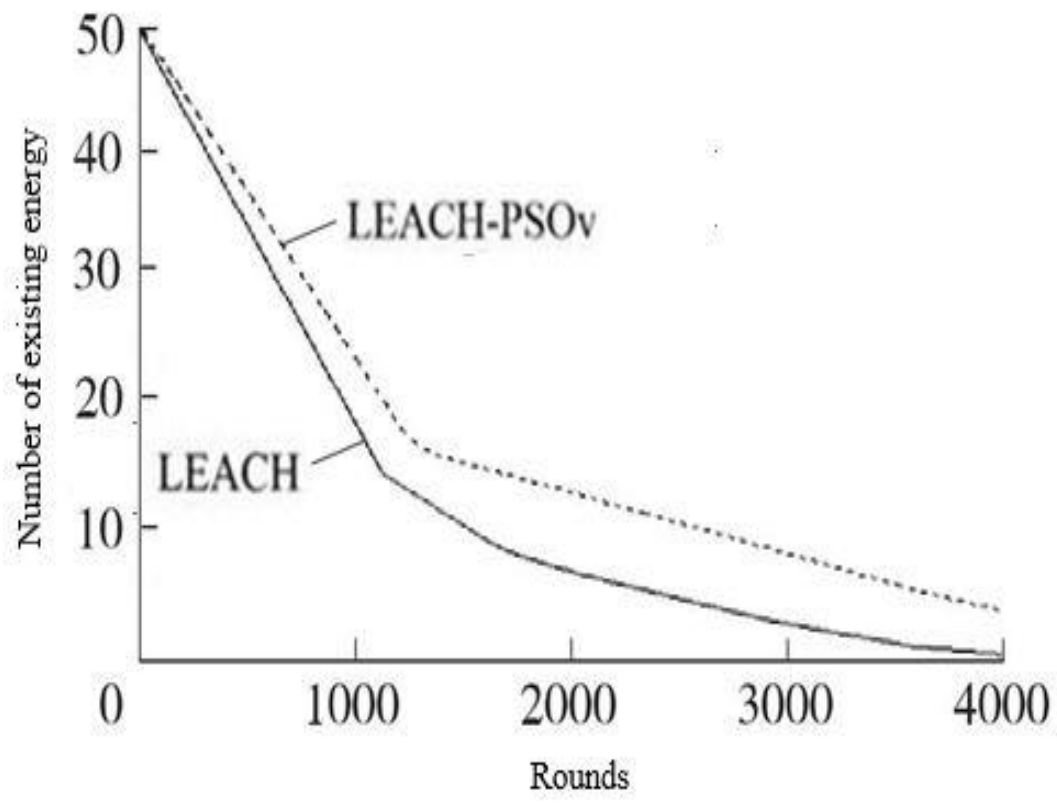

Figure 5. Existing Energy of Network in both LEACH and LEACH-PSOv Algorithm

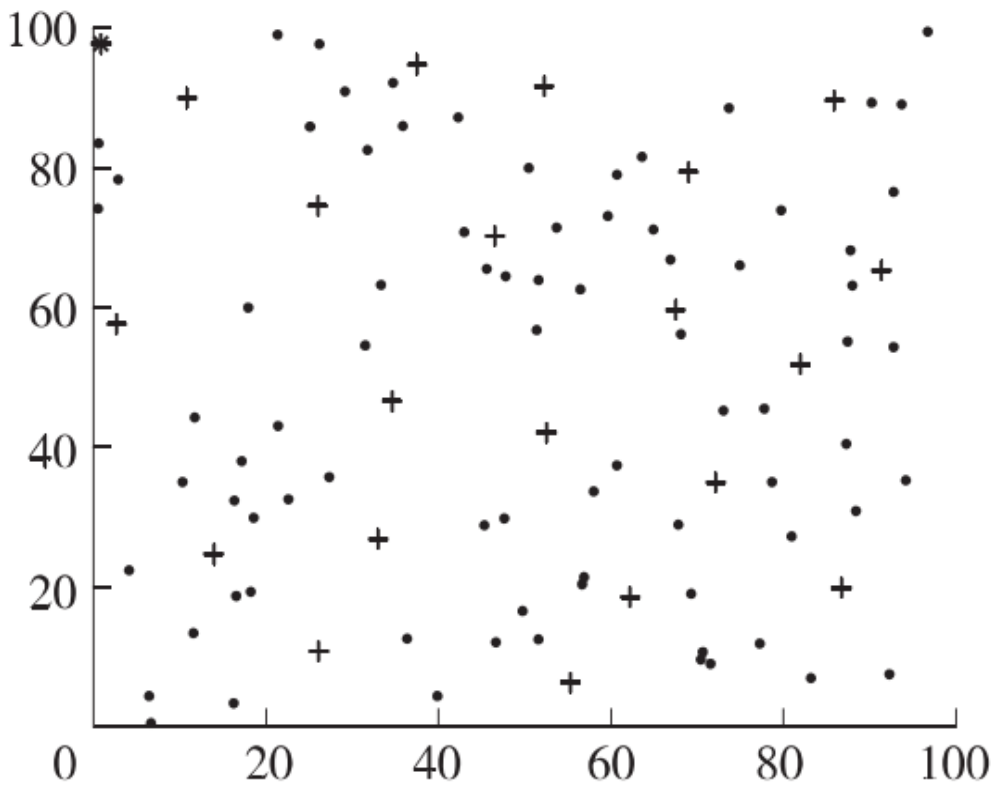

Figure 6. Nodes Distribution Base on LEACH 


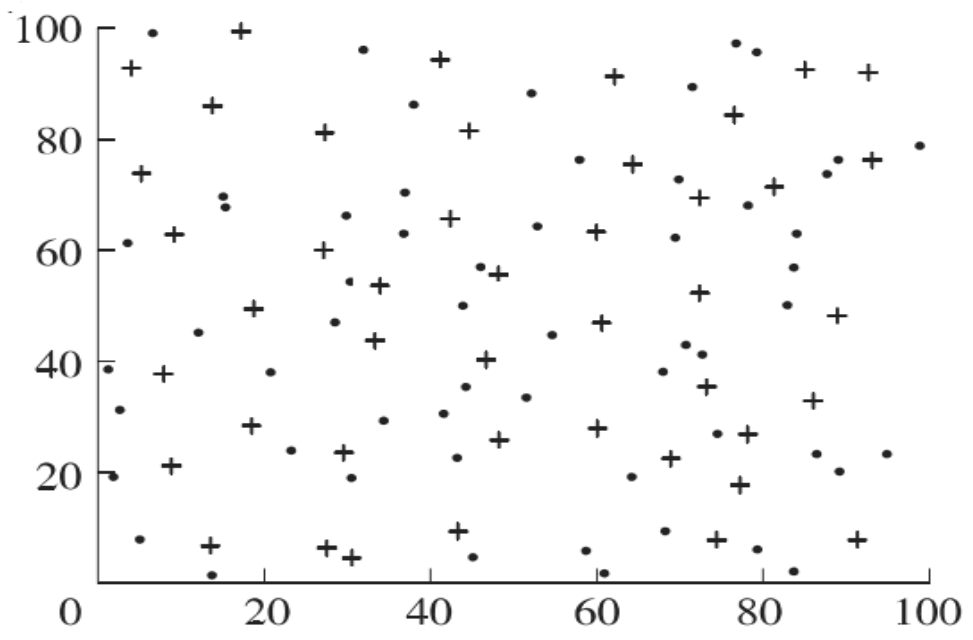

Figrue 7. Nodes Distribution Base on LEACH-PSOv

From Figure 5 shows the existing energy of network with the number of rounds growing. At the beginning of the calculation, the energy consumption with both algorithm differs little. As the number of rounds grows bigger and bigger, energy left in the network with LEACHPSOv algorithm becomes more than that with LEACH algorithm. After 1300 rounds, the difference of the network between the two algorithms becomes steady.

Figure 6 and Figure 7 showed the nodes distribution of the network in 1500th round with LEACH and LEACH-PSOv, respectively. "+" represents the live node, and "॰" represents dead node. We can see that the number of dead nodes is 81 in figure 6 while the number of that is 58 in figure 7. It is obviously that the LEACH-PSOv algorithm can provide more alive nodes. The operation and reliability performance has been greatly improved. In leach algorithm, when it is at 1500th round, $80 \%$ of the total nodes have been died, operation and reliability performance is quite worse.

\section{WSN Based Environmental Monitor System Design}

\subsection{Environmental Monitor System Design}

Environment monitor system the paper proposed is to monitor the temperature and humidity of the specified area, such as vagetable green house, and so on. Figure 8 shows the structure of the monitoring system. The system is composed of nodes (sensors), base station (sink node), GSM network, Remote monitor and computer. Sensors communicate with base station with LEACH-PSOv. Base station transfer messages to remote monitor through GSM network, and monitor transmit the data to computer.

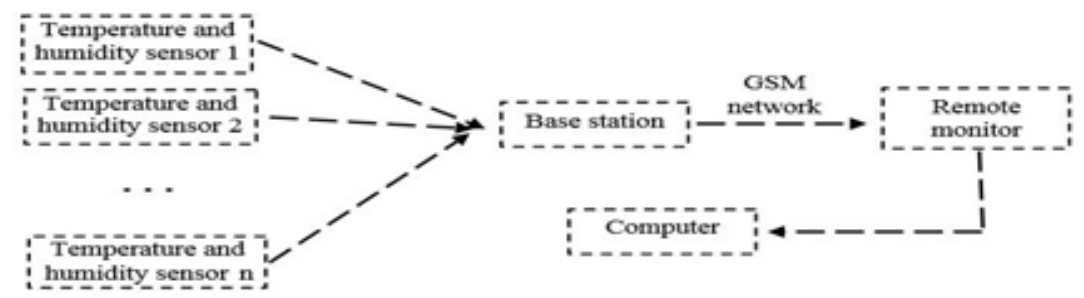

Figure 8. Structure of Environmental Monitoring System 


\subsection{Node Design}

Node is unit to collect temperature and humidity data and send them to base station. Temperature sensor and humidity sensor have been used in the nodes. Data transmission use MCU and Antenna. The node structure is showed in Figure 9.

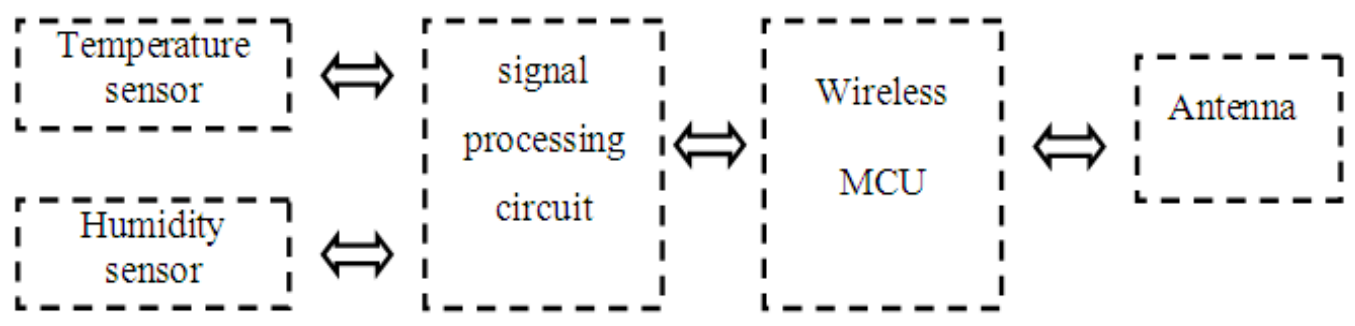

Figure 9. Structure of Node

\subsection{Base Station Design}

The desgn of base station includes wireless MCU, control circuit data transimission unit. Wireless MCU is used to colloct data from each node and send them to master control MCU. The master control MCU process the data and send the processed data to remote monitor. Figure 10 is the structure of base station.

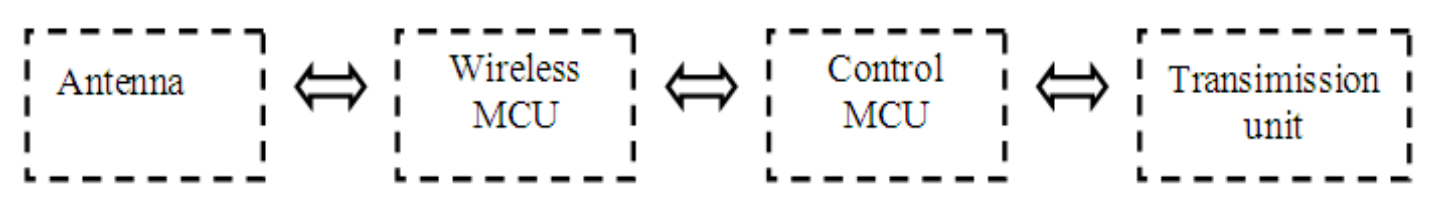

Figure 10. Structure of Base Station

\subsection{Temperature and Humidity Signal Process Circuit}

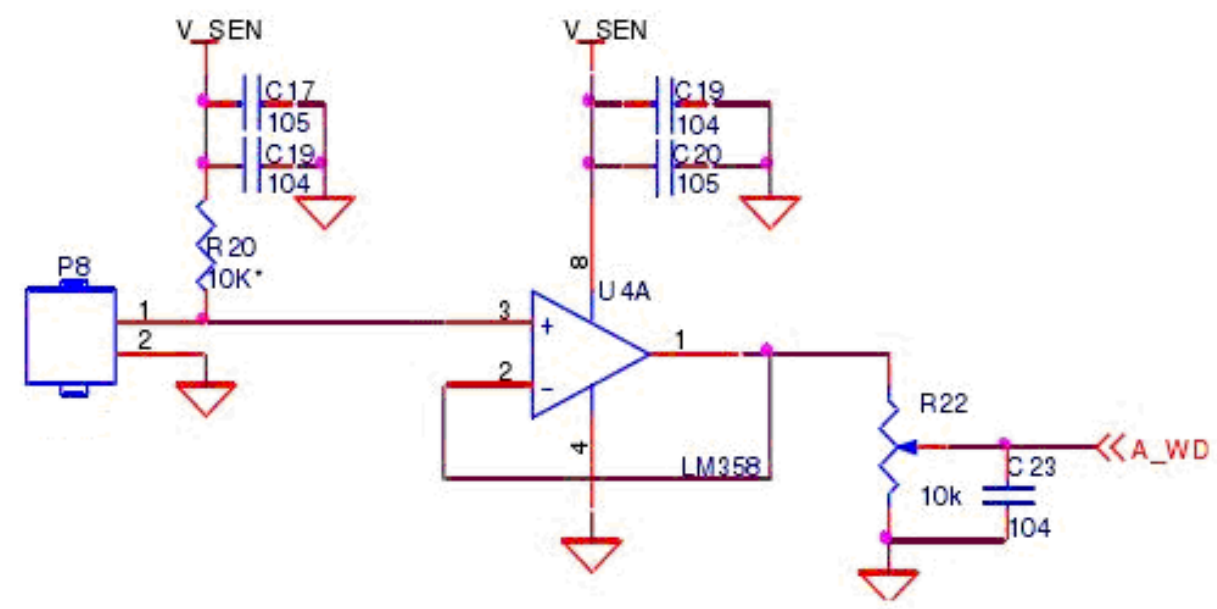

Figure 11. Temperature Signal Process Circuit 
The sensor of temperature and humidity sensor use cS1101 which is produced by Crossbow company in America. Sensor to monitor temperautre is thermister and sensor to monitor humidity is humidity-sensitive resistor, essentially. The range of temperature range is from $-40{ }^{\circ} \mathrm{C}$ to $70{ }^{\circ} \mathrm{C}$, while the humidity sensor range is between $0 \mathrm{Cbar}$ and $200 \mathrm{Cbar}$. Precision of both sensors is $\pm 5 \%$. Due to the the temperature and humidity sensor of eS1101 is resistance type, precision resistor is with the series of sensor to convert the resistance to voltage. The signal would be processed by LM358 and sent to ADC of CC2530. The circuit of temperature and humidity signal processes are showed with Figure 11 and Figure 12.

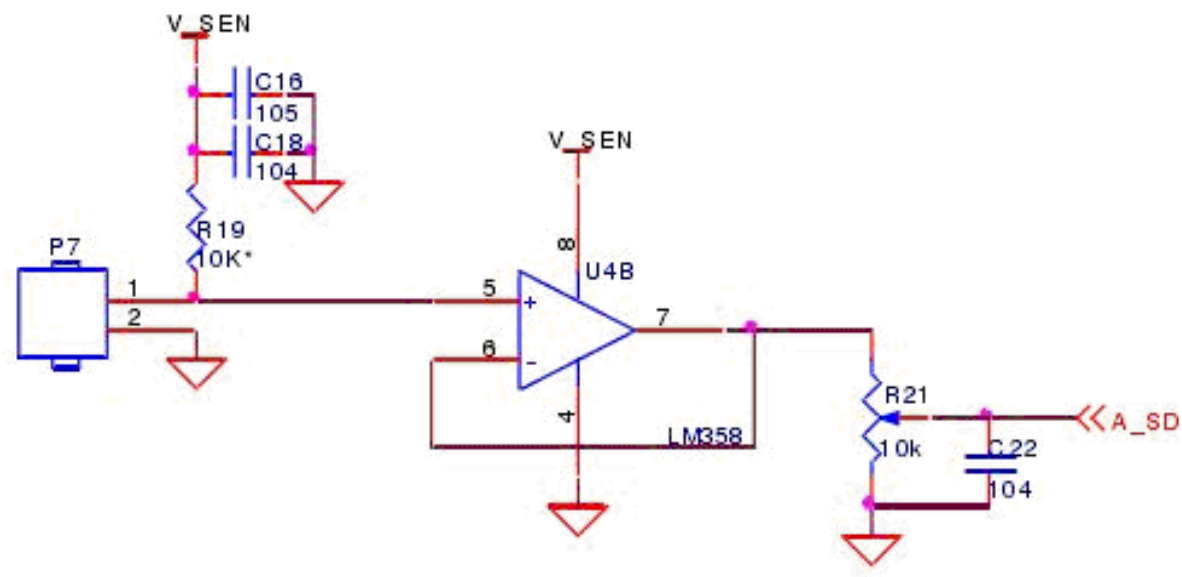

Figure 12. Humidity Signal Process Circuit

\section{Conclusion}

The paper, we discuss the LEACH protocol and analyzed it. Followed by an overview of LEACH protocol implements, then we proposed a new algorithm of LEACH protocol called LEACH-PSOv.

We have concentrate on comparison of LEACH and improved LEACH-PSOv. LEACHPSOv proposed in the paper from the aspect of energy balance of each node in the network. Firstly, the whole network would be divided in more than one sub clusters. The method can avoid the transmission of message from a long way and effectively save energy of each node. Secondly, cluster head node and vice head node would be elected based on energy and distance to balance the energy consumption. The algorithm can extend the lifetime and improve the efficiency of the network.

From the simulation results, we can draw a number of conclusions as following: (1) the first node in network with LEACH-PSOv died later than that in LEACH about 300 rounds, this means the energy consumption is quite balanced; (2) In 1500th round, number of dead nodes in improved algorithm is smaller than that in LEACH.

Based on the improved algorithm, environmental monitoring system has been designed. Results of the simulation show that LEACH-PSOv is an effective algorithm in WSN.

\section{Acknowledgement}

This study was supported by the projects of Application and Study on Greenhouse environment monitoring system based on WSN (Quzhou Science and Technology Bureau, No. 20121046) and Design of Gas pollution source Detection System in chemical industry based on WSN(Zhejiang education department, No. Y201327392) and Design and Implementation 
of University Laboratory Management Platform based on IOT(Students in Zhejiang Province Technology Innovation Project, No. 2013R427007).

\section{References}

[1] J. K. Hart and K. Martinez, "Environmental Sensor Networks: A revolution in the earth system science", Earth-Science Reviews, vol. 78, (2006), pp. 177-191.

[2] G. Werner-Allen, K. Lorincz, M. Welsh, O. Marcillo, J. Johnson, M. Ruiz and J. Lees, "Deploying a Wireless Sensor Network on an Active Volcano. IEEE Internet Computing, 10(2):18-25. (2006)

[3] I. Vasilescu, K. Kotay, D. Rus, M. Dunbabin and P. Corke, "Data collection, storage, and retrieval with an underwater sensor network", Proceedings of the 3rd international conference on Embedded networked sensor systems, (2005), pp. 154-165.

[4] J. up Martinez, K. Hart and J. K. Ong, "Deploying a Wireless Sensor Network in Iceland”, Lecture Notes in Computer Science, Proc. Geosensor Networks, vol. 5659, (2009), pp. 131-137.

[5] M. Ghanem, Y. Guo and J. Hassard, "Air Pollution Monitoring and Mining Based on Sensor Grid in London", Sensors, vol. 8, no. 6, (2008), pp. 3601.

[6] Anastasi, "A Comprehensive Analysis of the MAC Unreliability Problem in IEEE 802.15.4 Wireless Sensor Networks", vol. 7, no. 1, (2010), pp. 52-65.

[7] S. Pruter, G. Moritz, E. Zeeb, F. Golatowski and D. Timmermann, "Applicability of Web Service Technologies to Reach Real Time Capabilities", 11th IEEE International Symposium on Object Oriented Real-Time Distributed Computing (ISORC), (2008), pp. 229-233.

[8] Y. Liu, Y. Zhao and J. Gao, "A New Clustering Mechanism Based On LEACH Protocol”, 2009 International Joint Conference on Artificial Intelligence, (2009), pp. 715-718.

[9] B. Abu Bakr and L. Lilien, "A Quantitative Comparison of Energy Consumption and WSN Lifetime for LEACH and LEACH-SM", 2011 31st International Conference on Distributed Computing Systems Workshops, (2011), pp. 182-191.

[10] H. Junping, J. Yuhui and D. Liang, “A Time-based Cluster-Head Selection Algorithm for LEACH”, 2008 IEEE, (2008), pp. 1172-1176.

[11] A. Ahlawat and V. Malik, "An Extened Vice-Cluster Selection Approach to Improve V Leach Protocol in WSN", 2012 Third International Conference on Advanced Computing \& Communication Technologies, (2013), pp. 236-240.

[12] W. Luan, C. Zhu, B. Su and C. Pei, "An Improved Routing Algorithm on LEACH by Combining Node Degree and Residual Energy for WSNs", IOT Workshop 2012, CCIS 312, (2012), pp. 104-109.

[13] P. Xue-feng and L. La-yuan, "Design of an Energy Balanced Based Routing Protocol for WSN", 2011 IEEE, (2011), pp. 366-369.

[14] R. Kishore Kodali and N. Sarma, "NVS Energy Efficient Routing Protocols for WSN's", 2013 International Conference on Computer Communication and Informatics (ICCCI -2013), (2013).

[15] F. Zhao, Y. Xu, R. Li and W. Zhang, "Improved Leach Communication Protocol for WSN", 2012 International Conference on Control Engineering and Communication Technology, (2012), pp. 700-702.

[16] J. Xu, N. Jin, X. Lou, T. Peng, Q. Zhou and Y. Chen, "Improvement of LEACH protocol for WSN", 2012 9th International Conference on Fuzzy Systems and Knowledge Discovery (FSKD 2012), (2012), pp. 21742177.

[17] W. Wei, P. Shen, L. Zhang, H. Xu, J. Song, W. Zhang and W. Wang, "LEACH-Based Energy-Conserved Improved Protocol for WSNs", International Journal of Digital Content Technology and its Applications (JDCTA), vol. 6, (2012), pp. 163-171.

[18] L. Han, "LEACH-HPR: An Energy Efficient Routing Algorithm for Heterogeneous WSN", 2010 IEEE, (2010), pp. 507-511.

[19] X. Long-long and Z. Jian-jun, "Improved LEACH Cluster Head Multi-hops Algorithm in Wireless Sensor Networks", Ninth International Symposium on Distributed Computing and Applications to Business, Engineering and Science, (2010), pp. 10-12.

[20] J. Kennedy and R. Eberhart, "Particle Swarm Optimization", Proceedings of IEEE International Conference on Neural Networks IV, (1995), pp. 1942-1948.

[21] Y. Shi and R. C. Eberhart, "A modified particle swarm optimizer", Proceedings of IEEE International Conference on Evolutionary Computation, (1998), pp. 69-73.

[22] R. Poli, "Analysis of the publications on the applications of particle swarm optimisation", Journal of Artificial Evolution and Applications, (2008), pp. 1-10.

[23] S. Mostaghim, Dept. of Electr. Eng., Paderborn Univ., Germany; Teich, J. Strategies for finding good local guides in multi-objective particle swarm optimization (MOPSO), Swarm Intelligence Symposium, (2003), pp. 26-33. 
[24] C. Coello and C. A. Mopso, "A proposal for multiple objective particle swarm optimization”, Evolutionary Computation, (2002), pp. 1051-1056.

[25] S. Kitamura, K. Mori, S. Shindo, Y. Izui and Y. Ozaki, "Multiobjective energy management system using modified MOPSO", vol. 4, (2005), no. 3497-3503.

\section{Authors}

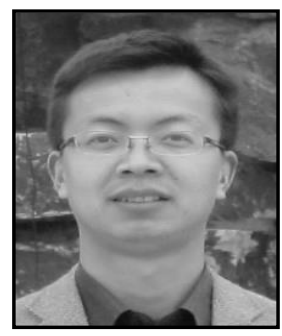

Xiajun Ding. He received his M.Sc. in Engineering (2005) from University. Now he is lecturer of Computer Science and Technology professionals University. His current research interests include different aspects of Wireless Sensor Networks and Intelligent information processing.

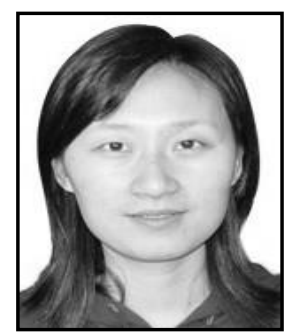

Xiaodan Jiang. She received her M.Sc. in Engineering (2009) from University. Now she is lecturer of Computer Science and Technology professionals, University. Her current research interests include different aspects of Wireless Sensor Networks and Artificial Intelligence.

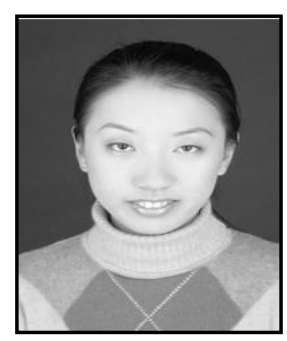

Lu Zhang. She received her M.Sc. in Engineering (2008) from University and she is now $\mathrm{PhD}$ from University. Now she is lecturer of Computer Science and Technology at College of Electrical and Information Engineering, University. Her current research interests include different aspects of Wireless Sensor Networks and Intelligent information processing.

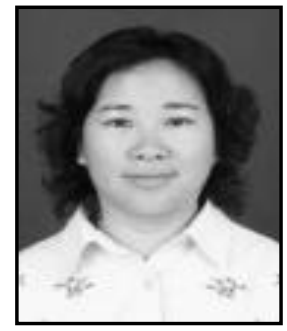

Shuangxia Han. She received her M.Sc. in Engineering (2005) from University. Now she is associate professor of Computer Science and Technology at College of Electrical and Information Engineering, University. Her current research interests include different aspects of Wireless Sensor Networks and E-commerce. 